\section{A COMPREHENSIVE CLINICAL AND GENOMIC CHARACTERIZATION OF LONG-TERM RESPONDERS RECEIVING IMMUNE CHECKPOINT BLOCKADE FOR METASTATIC NON-SMALL CELL LUNG CANCER}

'Paola Ghanem*, 'Joseph Murray, ${ }^{2}$ Melinda Hsu, 'David Ettinger, 'Josephine Feliciano, 'Patrick Forde, ${ }^{1}$ Christine Hann, ${ }^{1}$ Vincent Lam, ${ }^{1}$ Benjamin Levy, 'Julie Brahmer, ${ }^{1}$ Kristen Marrone. ${ }^{1} J o h n s$ Hopkins University, Baltimore, MD, USA; ${ }^{2}$ University Hospital Case Western Reserve, Cleveland, OH, USA

Background Five-year survival analyses of patients receiving immune checkpoint inhibitors (ICIs) for metastatic non-small cell lung cancer (NSCLC) have demonstrated continued clinical benefit compared to chemotherapy. ${ }^{1}{ }^{2}$ Our study aims at understanding and defining the unique clinical and genomic underpinnings of a durable response to ICI in advanced NSCLC.

Methods We conducted a retrospective case-control study using information abstracted from a Johns Hopkins IRBapproved database of NSCLC patients treated with an ICIcontaining regimen. We defined long-term responders (LR) as patients who have achieved an overall survival (OS) of at least 3 years. We identified a comparison arm (C) of patients whose OS was less than a year. Univariate and multivariate analyses of the clinical and molecular characteristics were conducted between the LR and C groups using IBM Statistical Package for Social Sciences version 25.

Results A cohort of 89 patients were included; 41 patients as LR and 48 as C. Mean duration of ICI was 21.6 months and 3.5 months for LR and C, respectively. On univariate analysis, there was no statistically significant difference in age, sex, race, histology or treatment characteristics between arms. However, ECOG performance status (PS) of $2(p=0.011)$ and evidence of liver metastases were independently associated with a shorter response to ICI $(\mathrm{p}=0.012)$. Increased PD-L1 expression was significantly associated with likelihood of LR status $(\mathrm{OR}=1.018, \mathrm{p}=0.027) .65 .9 \% \quad(\mathrm{n}=27)$ of $\mathrm{LR}$ patients developed an immune-related adverse event (irAE), of which 20 patients required discontinuation of therapy. In the $\mathrm{C}$ arm, $16.7 \%(\mathrm{n}=8)$ of patients developed an irAE of which 4 patients required discontinuation. On multivariate analysis, including age, sex, race, ethnicity, smoking status, BMI, PS, liver and brain metastases as well as the presence of common oncogenic molecular alterations, PS of $<2$ was statistically significantly associated with an $O S \geq 3$ years $(O R=16.7, p=$ 0.017). Molecular profiling was completed in 53 patients $(\mathrm{LR}=29, \mathrm{C}=24)$. Common molecular alterations were identified in 28 out of 53 patients ( $\mathrm{LR}=16, \mathrm{C}=12)$. KRAS mutation was assessed in 34 patients $(\mathrm{LR}=16, \mathrm{C}=18)$ and was associated with LR status versus $C$ (Fisher's exact test value $\mathrm{p}=0.0386$ ).

Conclusions Our retrospective study assessing multiple clinical and molecular determinants of patients with long-term response to immune checkpoint blockade, identified PS at diagnosis and KRAS mutation status to be associated with long-term response. Current efforts are ongoing to interrogate more deeply molecular features of LR, as well as their relationship to clinical aspects of a sustained benefit from ICI in NSCLC.

\section{REFERENCES}

1. . Borghaei $\mathrm{H}$, et al. Five-year outcomes from the randomized, phase III trials checkMate 017 and 057: nivolumab versus docetaxel in previously treated non-smallcell lung cancer. Journal of Clinical Oncology 0, JCO.20.01605, doi:10.1200/ jco.20.01605 (2021)
2.. Reck $M$, et al. Five-year outcomes with pembrolizumab versus chemotherapy for metastatic non-small-cell lung cancer with PD-L1 tumor proportion score $\geq 50$. J Clin Oncol 39:2339-2349, doi:10.1200/jco.21.00174 (2021)

Ethics Approval The retrospective case-control study has obtained ethics approval from the Institutional Review Board at the Johns Hopkins School of Medicine.

http://dx.doi.org/10.1136/jitc-2021-SITC2021.296 